\title{
DILEMAS SOCIALES DE LA PESCA EN EL PACÍFICO COLOMBIANO: UN ANÁLISIS DESDE LA TEORÍA DE JUEGOS*
}

\author{
JOSÉ SANTIAGO ARROYO MINA** \\ PONTIFICIA UNIVERSIDAD JAVERIANA, CALI
}

Recibido/ Received/ Recebido: 08/10/2011 - Aceptado/ Accepted / Aprovado: 03/02/2012

\begin{abstract}
Resumen
Este documento centra su análisis en el estudio de las normas sociales de la población dedicada a la extracción del recurso natural pesca, en el pacífico colombiano. Para tal efecto, se plantea un juego no cooperativo con información completa imperfecta entre la comunidad de pescadores de la zona y propietarios de los centros de acopio, que responde a los supuestos de la elección racional de los agentes, esbozados en la escuela neoclásica. El aporte de este documento consiste en caracterizar y modelar el fenómeno del intercambio directo de especies provenientes de una pesca ilegal; dentro un marco metodológico de teoría de juegos, y contrastar las condiciones de un Equilibrio de Nash para tal interacción estratégica.
\end{abstract}

Palabras clave: recursos naturales, pesca, racionalidad, equilibrio de Nash.

\section{FISHING SOCIAL DILEMMAS IN COLOMBIAN PACIFIC OCEAN: A ANALYSIS FROM GAME THEORY}

\begin{abstract}
This paper focuses on the social rules study of the population that works in fishing activities in Colombian pacific ocean. For such effect, a non-cooperative game is established with imperfect complete information among the zone of fish producers community and owners of collection centers, which responds to the assumptions of agents' rational choice, from neoclassic school. The contribution of this paper is to characterize and model the phenomenon of direct trade of species from illegal fishing; within a methodological framework of game theory, and contrast the conditions of a Nash Equilibrium for such strategic interaction.
\end{abstract}

Keywords: fishing, natural resource, rationality, Nash equilibrium

* Artículo resultado del proyecto de investigación "Normas sociales de la población dedicada a la pesca en el pacífico colombiano: una exploración desde el laboratorio económico", desarrollado por el Grupo Microeconomía Aplicada y Métodos Experimentales, MIMEX, categoría C de Colciencias. El proyecto fue financiado por la Pontificia Universidad Javeriana Cali y World Wildlife Fund (WWF Colombia).

** Profesor Departamento de Economía y Director del Grupo MIMEX, de la Pontificia Universidad Javeriana Cali. Estudiante de Doctorado en Economía de los Recursos Naturales y Desarrollo Sustentable, Universidad Nacional Autónoma de México. Magíster en Economía Regional, Universidad Austral de Chile. Correo electrónico: jarroyo@javerianacali.edu.co 


\title{
DILEMAS SOCIAIS DA PESCA NO PACÍFICO COLOMBIANO: UMA ANÁLISE DESDE A TEORIA DE JOGOS
}

\section{Resumo}

\begin{abstract}
Este documento centra sua análise no estudo das normas sociais da população dedicada à extração do recurso natural pesca, na região do pacífico colombiano. Para isto, propõe-se um jogo não cooperativo, com informação completa imperfeita, entre a comunidade de pescadores da zona e os proprietários dos centros de provisão. Pretende-se que tal jogo responda aos supostos da escolha racional dos agentes, esboçados na escola neoclássica. A contribuição deste documento consiste em caracterizar e modelar o fenômeno do intercâmbio direto de espécies provenientes de uma pesca ilegal e contrastar as condições de um Equilíbrio de Nash para tal interação estratégica, dentro um marco metodológico de teoria de jogos.
\end{abstract}

Palavras chave: recurso natural, pesca, racionalidade, equilíbrio de Nash

Arroyo, J. (2012) Dilemas sociales de la pesca en el pacífico colombiano: un análisis desde la teoría de juegos. En: Revista de la Facultad de Ciencias Económicas de la Universidad Militar Nueva Granada. rev.fac.cienc.econ, $\mathrm{XX}(1)$

JEL: $I 12, K 14, K 42$.

\section{Introducción}

Existen diferencias importantes en lo que concierne a necesidades, capacidades y situaciones propias, de aquellas comunidades que habitan el casco urbano, respecto a las que habitualmente se ubican en la parte rural y costera de los países latinoamericanos. Los gobiernos centrales no han sido capaces de distinguir este tipo de diferencias, que originan desigualdad social, toda vez que, en realidad, ellos normalmente se preocupan por alcanzar sus objetivos de política de corto plazo, tal y como lo señala Elbers et al. (2004).

A partir de lo anterior, para este artículo resulta importante señalar que una causa del problema de pes- ca ilegal ${ }^{1}$ que se origina en el pacífico colombiano ${ }^{2}$, se explica en buena medida por el nivel de desigualdad social existente dentro de la población que habita dicha zona ${ }^{3}$. En tal sentido, este documento centra su análisis en el marco teórico de las normas sociales de aquellas poblaciones expuestas al manejo de recursos de uso común, así como del componente institucional que rodea a tales poblaciones.

Para comprender de mejor forma el comportamiento de las poblaciones involucradas en el manejo de recursos de uso común, la teoría de juegos no cooperativos se convierte en una extraordinaria herramienta que facilita el desarrollo de las predicciones teóricas que puedan resultar de la interacción estra-

1 En concreto, se refiere a situaciones de pesca dentro de las fechas establecidas como vedas, pesca en zonas o áreas protegidas, pesca con artes (anzuelos, mallas, etc.) no aprobadas por el regulador, entre otras situaciones. Adicionalmente, las especies pertenecientes a la pesca, a las que se hace referencia en este documento y que pertenecen tanto al arte de pesca blanca (pesca de espinel: anzuelos), como de pesca roja (pesca de malla); son aquellas que se encuentran en riesgo de agotamiento en la zona de estudio. Algunas de ellas, son: Pargo, Sierra y Camarón Langostino.

2 La investigación estudia el comportamiento de los pescadores del Golfo de Tribugá (Choco), zona de fuerte influencia pesquera dentro del pacífico colombiano.

3 A modo de ejemplo, la cobertura en educación secundaria para la población que vive en varias regiones de la costa pacífica es del 62 $\%$, mientras que el resto de la nación presenta $75 \%$. En educación superior, la cobertura es de sólo el 14\% para el grupo en mención, mientras que es del $26 \%$ para el resto de la nación. La población afro-colombiana, que no presenta ningún tipo de seguridad social es del $51 \%$, mientras que igual tasa para el resto de la población es del $35 \%$. Adicionalmente, 7.285 afrocolombianos por cada 10.000 habitantes están en alto riesgo de malaria (DNP, 2004). 
tégica de tales poblaciones, tal y como lo advierte Ostrom et al. (1994); anotando por supuesto, que los resultados no pueden llegar a ser una generalización teórica.

Adicionalmente, no se puede obviar que la presencia de desigualdades sociales al interior de este tipo de comunidades, se refleja en mecanismos de supervivencia inapropiados que terminan atentando la estabilidad de los ecosistemas y la sostenibilidad de los recursos naturales.

A modo de ejemplo, la desigualdad social motiva la pesca ilegal. Según Cárdenas (2003) y Cárdenas et al. (2002), la exploración del bienestar de este tipo de comunidad, resulta ser un factor que está asociado a preferencias más o menos sociales dentro de la comunidad. En otras palabras, el comportamiento de la población rural que se dedica a la extracción de recursos naturales de uso común (pesca), encuentran que es más difícil dar solución a un "Dilema de los Comunes" por mecanismos endógenos.

En concreto, la pesca ilegal que existe en aquellas comunidades que habitan la costa del pacífico colombiano, se justifica parcialmente por los fallos en el diseño de una política pública que realmente este orientada a la corrección de las desigualdades sociales para las comunidades que habitan en tal zona. Sin embargo, Hatcher et al. (2000) señalan que no puede desconocerse que este tipo de comunidades presenta rasgos particulares a su etnia y cultura, que deben ser estudiados desde el enfoque de las normas sociales que influyen en la extracción y agotamiento del recurso, tal y como es la propuesta de este documento.

A partir de los argumentos planteados en los párrafos anteriores, este documento desarrolla un juego no cooperativo con información completa imperfecta, que refleja una interacción estratégica entre las comunidades de pescadores y propietarios de los centros de acopio, involucrando los incentivos económicos y las normas sociales, como factores relevantes de este tipo de agentes, al momento de tomar la decisión de participar en la pesca ilegal.

Para ello, lo aquí registrado se ordena de la siguiente forma. La sección 1 es esta introducción, luego se continúa presentando lo que se podría entender por normas sociales, a la luz de los principales enfoques teóricos que han venido estudiando tal característica. Posteriormente se registra la interacción estratégica derivada del comportamiento de los agentes envueltos en la extracción de pesca ilegal, así como las preferencias presentes en el juego, la secuencia de las decisiones, la definición de estrategias y los posibles equilibrios a los que se llega en dicha interacción. Finalmente, el documento presenta una cuarta sección con algunos comentarios finales y reflexiones en torno al tema.

\section{Marco de referencia}

Para estudiar las normas sociales de la población del pacífico colombiano que está dedicada a la pesca ilegal, tal como lo señala Ehrlich (1996), es necesario suponer que los agentes (en este caso, comunidades de pescadores de la zona y propietarios de los centros de acopio) se comportan de forma racional ${ }^{5}$. A continuación, los teóricos fundamentales de las normas sociales, que hacen énfasis en la importancia del contexto que envuelve a las comunidades que se ubican en zonas de explotación de recursos de uso común.

\subsection{Recursos de Uso Común}

Siguiendo lo registrado en Ostrom et al. (1994), los recursos de uso común son un conjunto de situaciones en donde los individuos están involucrados en decisiones que resultan ser una sumatoria de eventos

4 El aporte de Hardin (1968) muestra que si nadie se interesa en las consecuencias de agotar el recurso, ni que aumente el costo, entonces se refrendará la famosa "tragedia de los comunes"; es decir, se tendrá un problema de acceso libre.

5 Para entender lo que en este documento se entiende por racionalidad, basta con recordar el trabajo pionero de Becker (1968), en donde se argumenta que el individuo al elegir entre las actividades legales e ilegales, toma una decisión racional teniendo en cuenta sólo los incentivos económicos. En otras palabras, se sugiere que la pesca al superar los límites óptimos de sustentabilidad del recurso natural, debería ser tratada como una actividad ilegal y esto es conocido por los pescadores. 
repetidos, que a su vez están basados en decisiones individuales que terminan repercutiendo en efectos sociales al interior de la comunidad. Es decir, muchas comunidades son responsables de una $\mathrm{u}$ otra manera del uso y manejo de recursos comunitarios entre los que se incluyen los bosques, los manglares, los recursos pesqueros, las microcuencas, y todos los demás recursos ambientales a los cuales tiene acceso la comunidad y de los que se obtienen beneficios monetarios y no monetarios en términos de los bienes y servicios ambientales que prestan (Cárdenas et al., 2003).

En tal sentido, señala Ostrom (2000) que este tipo de bienes se definen como recursos de uso comunitario. Los recursos de uso comunitario tienen dos características: la excluibilidad y la sustractibilidad, por lo que no pueden ser clasificados ni como bienes privados puros, ni como bienes públicos puros, y de ahí la importancia de que sean analizados conceptual y empíricamente de manera particular. La excluibilidad se refiere a no poder limitar el libre acceso pues esto resultaría muy costoso, es más, estos recursos suelen ser lo suficientemente grandes como para permitir su utilización de manera simultánea por muchos usuarios. La sustractabilidad, se explica en la medida en que cada usuario al extraer recursos puede sustraer de bienestar a los otros usuarios, ya que cada unidad extraída de un recurso reduce la posibilidad de otros usuarios de beneficiarse del ecosistema.

A modo de complemento, Cárdenas et al. (2003) indica que una de las propiedades más importantes de este tipo de recursos, es el tener inserto un dilema entre lo colectivo y lo individual, ya que los usuarios de este tipo de recursos no pueden disponer de unidades de recurso que ya han sido extraídas por otros, de la misma manera tampoco, se puede excluir a otros de las mejoras que se le hagan al re- curso; todos en la comunidad se verán beneficiados por estas mejoras. Por lo tanto, muchas personas tendrían incentivos privados para disfrutar de estos beneficios sin sacrificar nada a cambio, esta situación es la que Ostrom (1990) define como "el gorrón (free-rider)" y que en concreto no es otra cosa que el oportunista.

\subsection{Fundamentales teóricos de las normas sociales}

Según Ostrom et al. (1994) y Ostrom (2000), las comunidades involucradas en el manejo de recursos de uso común, presentan una serie de normas sociales que generalmente las caracteriza. Para ello, Ostrom (2000) define ocho principios de diseño que enmarcan este tipo de normas ${ }^{6}$. Como un complemento teórico de lo expuesto por Ostrom (2000), se encuentran los aportes de Harsanyi (1955) quien sugiere un camino para superar las limitantes del teorema de la imposibilidad de Arrow ${ }^{7}$.

Para Harsanyi (1955), el comportamiento cardinal de las preferencias de los agentes, basado en comparabilidad interpersonal, permite pensar en la existencia de un juez neutral (espectador imparcial), que teniendo en cuenta unas preferencias éticas, formula ciertos juicios de valor acerca del mejor ordenamiento social; es decir, aquel que favorezca una mayor justicia y nivel de bienestar para todos los individuos.

En tal sentido, Casas et al. (2003) afirman que las bondades del postulado de Harsanyi (1955) radican en que cualquier persona puede ocupar la figura de juez y de esta forma, al contar con igual probabilidad de ocupar cualquier posición derivada de su elección, buscará maximizar su utilidad esperada a la hora de optar por una determinada norma o sistema social.

De otra parte, dentro de los referentes teóricos de las normas sociales, no se puede pasar por alto lo regis-

6 Los principios son: Límites claramente definidos; Congruencia entre las reglas de apropiación y de provisión y las condiciones locales; Acuerdos de elección colectiva; Supervisión; Sanciones graduales; Mecanismos de solución de conflictos; Reconocimiento mínimo de los derechos de la organización; Actividades complementarias. Ostrom (2000) advierte sobre aquella situación de aplicación de los principios: "así este diseño haya sido adoptado, ello no necesariamente significa que se tengan comunidades eficientemente autogestionarias".

7 Arrow (1951) encontró incompatible las condiciones que posibilitarían la agregación de utilidades individuales en una función de bienestar social. 
trado en Sen (1988), toda vez que su enfoque es pionero en la deducción de preferencias sociales a través de las preferencias individuales. Esta situación, le permite a Sen (1988) encontrar el estado social resultante de las elecciones colectivas, específicamente le permite ordenar y evaluar estados sociales a partir de la construcción de indicadores de bienestar ${ }^{8}$, que a su vez requieren necesariamente de comparaciones interpersonales para estudiar las consecuencias distributivas (pobreza, desigualdad, etc.) de distintas sociedades.

Adicional a los planteamientos anteriormente referenciados, aparece la propuesta de Cárdenas \& Ostrom (2004), respecto a los ejercicios experimentales que se centran en la definición de distintas normas sociales, que a su vez contribuyen en posibles soluciones para aquellas situaciones de explotación de recursos naturales de uso común. De forma particular, Cárdenas \& Ostrom (2004) sugieren que los resultados de experimentos económicos de los arreglos organizados por ciudadanos para gobernar exitosamente los recursos de agrupación común, muestran que los grupos que pueden comunicarse reiteradamente, terminan estableciendo un conjunto de normas sociales que les permite alcanzar resultados prácticamente óptimos, en vez de hacer una sobreexplotación desmesurada del recurso natural.

Baland \& Platteau (1997) indican que establecer normas sociales ocasionan dos tipos de efectos en el comportamiento individual de los agentes: limitan sus decisiones y pueden llegar a formar preferencias. Respecto a limitar decisiones, basta con recordar el principio de reciprocidad que sugiere Sugden (1984) y Baland \& Platteau (1997), para quienes los dilemas sociales pueden llegar a resolverse bajo opciones de cooperación que sigan el camino de una norma reciproca entre el no oportunismo al interior de la comu- nidad. En concreto, si todos los jugadores siguen y respetan esta norma, se puede llegar a un óptimo social.

Para el caso particular de recursos de uso común, Bowles \& Gintis (2002) muestran que existe un efecto importante en los comportamientos pro-sociales que se definan en cada comunidad. Lo que sugiere tal argumento, es que los individuos pueden llegar a tener distintas motivaciones intrínsecas en sus decisiones $y$ esto puede resultar en situaciones que en algunos momentos podrían ser predecibles, pero en otros no.

De igual forma, Bowles (2008) indica que la interacción estratégica entre individuos con un comportamiento centrado en preferencias pro-sociales, favorece la obtención de un óptimo social, toda vez que estos se convierten en motivadores y promotores que incentivan un comportamiento pro-social, en este caso, a favor de la sustentabilidad de los recursos de uso común.

Finalmente, resulta importante referenciar los aportes teóricos de una elección discreta con interacción social, tal y como lo señalan Brock \& Durlauf (2001). Para ellos, por interacción social se entiende que la utilidad o pago que un individuo recibe de una acción dada, depende directamente de la elección de otros que están en el grupo de referencia del individuo. Los resultados alcanzados por Brock \& Durlauf (2001) resaltan que los individuos se identifican con su grupo de referencia, lo que demuestra cómo que el efecto de la utilidad conjunta es suficientemente grande y las decisiones son no-cooperativas; por tanto, existen múltiples equilibrios con comportamiento promedio, para este tipo de individuos.

\section{Modelo 9}

Con la intención de aportar a la comprensión del estudio de las normas sociales de la población de-

8 Para Sen (1988), el bienestar es un ejercicio de valoración y cuando se hace alusión a tal concepto, se hace entonces referencia a aspectos como la capacidad, las oportunidades, las ventajas, entre otros elementos imposibles de cuantificar (Nussbaum \& Sen, 1996).

9 El modelo teórico aquí presentado y que a su vez resulta ser una adaptación de lo registrado en Arroyo et al. (2009), contribuye de alguna manera, en la formulación de respuestas para el problema del agotamiento de los recursos naturales. En tal sentido, trabajos como los de Trisak (2005), Jensen \& Vestergaard (2000) y Milliman (1986), pueden ser de interés para todos los lectores apasionados en la teoría de juegos y sus aplicaciones teóricas hacia los recursos naturales. 
dicada a la pesca ilegal en el pacífico colombiano, a continuación se presenta la definición de las posibles estrategias que pueden estar presentes en una interacción estratégica entre pescadores de la zona en mención y propietarios de los centros de acopio.

\subsection{Definición de estrategias}

El modelo sugiere que la utilidad total que deriva un individuo al tomar una decisión de captura o compra de pescado proveniente de niveles de extracción que atentan contra la sostenibilidad del recurso de uso común (pesca ilegal), depende de cómo ésta se vea afectada por la utilidad privada y la utilidad social ligada a su elección.

La utilidad privada va a depender de los costos asociados a la ilegalidad y los costos asociados a la actividad propiamente dicha ${ }^{10}$. Hatcher et al. (2000), sugieren que la decisión de obedecer o violar la ley que establece el regulador ambiental, depende principalmente del nivel de gastos monetarios esperados y de las posibles ventajas económicas en las que el pescador cree o asume que puede incurrir. Por ello, las decisiones de regulación externa normalmente tienden a enfocarse sólo en la fuerza de disuasión, es decir, el regulador envía una señal al pescador ilegal en términos de aumentos de sus gastos monetarios de violación.

A partir de lo anterior, Hatcher et al. (2000) afirman que los modelos de comportamiento de cumplimiento de pesca sustentable, deben tener en cuenta factores de influencia social, valores morales, y por supuesto, la legitimidad percibida por los pescadores en términos de la regulación externa. Así las cosas, la utilidad social va a depender de lo que en promedio el individuo espera que haga su grupo de referencia ${ }^{11}$.
Considerando que el modelo aquí planteado corresponde a un juego no cooperativo de información completa, en donde se tienen ya especificados los jugadores, sus estrategias y los pagos asociados a dichas estrategias, se puede resumir la estructura del juego, de la siguiente forma:

$$
\mathrm{L}=[\mathrm{P}, \mathrm{D}]
$$

Donde $L$ denota el conjunto de jugadores que participa en el intercambio, la letra $P$ se utiliza para representar a los pescadores que capturan el recurso de forma ilegal y la $D$ para los dueños o propietarios de los centros de acopio. Estos jugadores cuentan con diferentes estrategias, que serán expresadas a través de los conjuntos $S^{P}$ y $S^{D}$ para pescadores y dueños o propietarios, respectivamente.

$$
\begin{gathered}
\mathrm{SP}=[\mathrm{np}, \mathrm{pi}, \mathrm{pl}] \\
\mathrm{SD}=[\mathrm{nc}, \mathrm{c}]
\end{gathered}
$$

Las estrategias del pescador son $n p$, que significa no pescar de forma ilegal; pi, pescar de forma ilegal; o $\mathrm{pl}$, pescar de forma legal. Por otra parte, los dueños o propietarios de los centros de acopio tienen como estrategias no comprar especies provenientes de capturas ilegales nc, o comprar, denotado por $c$.

Si los individuos, al hacer un análisis costo-beneficio encuentran que es mejor estar en la legalidad que comprometerse en este tipo de actividades ilegales, no entrarán en el juego del intercambio directo de especies provenientes de una extracción de pesca ilegal, situación que permitirá que su nivel de utilidad de realizar actividades ilegales sea normalizado a cero $^{12}$; así mismo ocurre con el dueño del centro de

10 Aquellos costos asociados a la ilegalidad están determinados por la probabilidad de castigo. En concreto, se refiere a la probabilidad de aprehensión de los equipos con que se realiza la extracción del recurso de uso común, así como a la posibilidad de sellamiento o cierre del centro de acopio. Mientras que, los costos asociados a la actividad propiamente dicha están determinados por todos los factores que afectan a los niveles de extracción y de compra del pescado, como: costos del combustible y modalidad de transporte, facilidad para conseguirlos, etc.

11 Debe tenerse en cuenta que cuando aquí se habla de interacción social se está pensando en grupo de influencia del agente, porque aunque para algunos individuos su grupo de influencia es la familia, para otros puede que sean los vecinos, los amigos del barrio, o como lo es en este caso, el resto de la comunidad de pescadores de la zona; es decir, se resalta el estatus como una variable de impacto en la decisión de los individuos.

12 Sin pérdida de generalidad, este valor podría ser cualquier cantidad monetaria inferior a la que le representa trabajar en la legalidad y que sería su salario de reserva, en caso de que este no se afecte por ningún otro factor. 
acopio, en cuyo caso escogerán las estrategias $n p ; n c$ para el pescador y el dueño, respectivamente.

A partir de la caracterización de jugadores y estrategias expuestas anteriormente, es posible construir una función de utilidad para cada jugador que representa los pagos asociados a las estrategias. En términos formales:

$$
\text { si np,SjD o SiP, nc1- } \gamma \mathrm{iU} \pi \mathrm{i}+\gamma \mathrm{iUDiP}+\mathrm{G} \gamma \mathrm{if} \text { si } \mathrm{SiP}, \mathrm{c}
$$

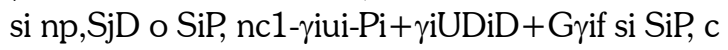

$$
\forall \mathrm{i}=\mathrm{np}, \mathrm{pi}, \mathrm{pl} \quad \mathrm{y} \quad \forall \mathrm{j}=\mathrm{nc}, \mathrm{c}
$$

En donde UPSP,SD y UDSP,SD son las utilidades esperadas de pescadores y dueños de centros de acopio, respectivamente ${ }^{13}$. Si el pescador opta por la estrategia np o el propietario del centro de acopio opta por nc, el pago será cero con lo cual el juego termina; mientras que, los pagos obtenidos al elegir una estrategia distinta, van a depender de la modalidad de extracción o compra, tal y como se observa en las ecuaciones (4) y (5).

A partir de la consideración del intercambio directo de especies provenientes de una extracción ilegal de pesca, en este documento se ha planteado la existencia de dos tipos de costos, aquellos asociados a la ilegalidad, que tienen que ver con la posibilidad de que los agentes sean castigados por la autoridad pública $^{14}$, y aquellos centrados en el castigo relativo a la pérdida de estatus social, que tienen que ver con la recriminación por algún miembro de su grupo de referencia, en función al quiebre de la norma social establecida, entiéndase extracción favorable en términos de sustentabilidad del recurso (pesca legal). Para destacar este último aspecto se ha utilizado la letra $G$ y para el costo asociado a la transacción propiamente dicha se utiliza la letra $C$. El costo asociado a la ilegalidad y que tiene que ver con el castigo por parte de la autoridad y la probabilidad de ser capturado, es descrito por:

riDiL

Este costo está compuesto por la probabilidad de ser capturado por la autoridad ${ }^{15} \mathrm{\gamma i}$ y el equivalente monetario del castigo recibido $\mathrm{DiL}^{16}$. La probabilidad de captura y el castigo dependen de la modalidad de intercambio ${ }^{17}$.

De otro lado, es interesante resaltar que en las ecuaciones (4) y (5) existe una variable denotada con la letra $G$, que es una variable dicotómica que toma los valores de "cero" si al agente (pescador o dueño del centro de acopio) no le importa el estatus y "uno" si

13 Tal y como lo sugieren Neumann \& Morgenstern (1947), se puede definir el nivel de utilidad esperado de un agente particular, como sigue: $\pi e=$ wi-ci-wL-pifi

Es decir, el retorno neto esperado $\pi$ e por participar en una actividad ilegal (en este caso extracción inapropiada del recurso natural de uso común: pesca), es igual al pago bruto por cometer el delito wi, menos el costo directo ci, menos los ingresos no percibidos de actividades legales wL (los cuales se conocen con certeza absoluta), menos el castigo ponderado por la probabilidad de ser capturado y castigado pifi. Es necesario resaltar que hay incertidumbre sobre los ingresos percibidos por actividades ilegales, por eso se incluye pi.

14 En este caso, por autoridad pública deben entenderse tanto reguladores (monitores) de los parques nacionales naturales (áreas protegidas), así como agentes de la policía.

15 Se supone que captura implica castigo, es decir, se da por sentado que si un individuo es capturado cometiendo un delito, en este caso pesca ilegal o compra de especies provenientes de tal pesca, debe ser castigado por la autoridad pública. A modo de ejemplo, aprehensión de los equipos (lancha, motor, mallas, anzuelos, entre otros) utilizados en tal extracción y/o sellamiento o cierre del centro de acopio.

16 Aunque se ha definido la variable $\mathrm{D}$ como el equivalente monetario del castigo recibido, cabe anotar que en este momento, la mayoría de legislaciones ambientales y de protección de los recursos naturales de la región latinoamericana, no tiene en realidad un control estricto en la aplicación de este tipo de sanciones. Por tal razón, este artículo hace mucho énfasis en el castigo social más que en el de la autoridad pública.

17 Dependiendo del entorno en que se desempeñe el pescador o propietario del centro de acopio, la probabilidad de captura será distinta. Además, se supone que el castigo va a depender de la cantidad de recurso natural de uso común que extraiga o compre el individuo. 
el estatus importa. Esta variable mide la importancia que pescador y dueño del centro de acopio dan al estatus (por estatus entiéndase imagen ante grupo de referencia), y $\lambda \mathrm{i}$ es una función de distribución, que denota la probabilidad de ser recriminado por el grupo de referencia.

Obsérvese que $\lambda$ está en función de f y f $\in 0,1$, dentro de este intervalo, el valor cero significa que el grupo de referencia tolera o convive con la ilegalidad, es decir, con la pesca ilegal y/o compra de las especies provenientes de tal extracción, mientras el valor uno, significa que el grupo de referencia no tolera comportamientos fuera de la ley.

Además del costo de la ilegalidad, se hace necesario evaluar qué sucede en caso de llevarse a cabo el intercambio directo de especies provenientes de una pesca ilegal de manera satisfactoria, ya que esto genera pagos distintos para cada agente (pescador y propietario del centro de acopio).

En concreto, el valor que arroja la pesca ilegal de especies para un pescador, será la cantidad de dinero que logre obtener de dicha transacción $1-\gamma \mathrm{iPi}-\mathrm{Ci}$, dependiendo de los costos $\mathrm{Ci}$ y del precio $\mathrm{Pi}$ de la modalidad de pesca; mientras que, el resultado que logra el propietario del centro de acopio al realizarse de manera satisfactoria la transacción es 1-yiui-Pi. En este caso, ui, se refiere a la dispocisión a pagar que pueden llegar a tener los agentes involucrados en el intercambio ilegal, en función de la dificultad de poder evadir los distintos controles presentes en la zona en que se realiza la transacción, la calidad y cantidad del tipo de especie comprada, y la fidelidad de los mismos en términos de la no denuncia durante la transacción.

\subsection{Condiciones para hallar equilibrios de Nash}

Luego de haber descrito los jugadores, las estrategias y la función de utilidad que permite definir los pagos; se plantean a continuación, un par de escenarios, en los que se tienen Equilibrios de Nash de un juego no cooperativo con información completa y en estrategias puras. Para lograr tales equilibrios, la combinación de estrategias debe satisfacer simultáneamente el siguiente problema de optimización:

$$
\text { UPSP, SD }
$$

$$
\text { UDSP, SD }
$$

Antes de mostrar los posibles equilibrios del juego no cooperativo entre pescadores y propietarios de centros de acopio, se debe destacar que el precio de la especie que ha sido capturada de forma ilegal, difiere por modalidad, ya que existe un cobro adicional en el intercambio que precisamente es originado dentro de este contexto de ilegalidad. En tal sentido, el propietario del centro de acopio debería estar dispuesto a pagar una tarifa adicional, toda vez que entiende que está obteniendo un beneficio derivado de tal ilegalidad; a modo de ejemplo, está obteniendo especies que en determinado horizonte temporal no podría obtener (fechas de veda), situación que le permite negociarlas a precios mayores.

Para desarrollar el análisis aquí propuesto, se plantean dos escenarios: en el primero de estos, la variable $G$ toma el valor "cero", lo cual significa que el estatus social no importa; en el segundo, dicha variable es "uno" significando que el estatus social sí importa.

\subsection{1. ¿Qué sucede cuando el status social no importa?}

En este escenario se analiza el comportamiento de los agentes cuando la variable $G=0$, y existe un perfecto conocimiento de las reglas que imperan en la zona ${ }^{18}$ en la que se realiza la pesca ilegal, lo cual hace que los riesgos asociados a este tipo de actividad, sean vistos como menores, por quienes optan por la modalidad de la ilegalidad. Adicionalmente, se asume que es más probable que un individuo sea capturado pescando de forma ilegal o comprando para el centro de acopio las especies que provienen de este tipo de pesca ilegal, con lo que $\gamma \mathrm{pl}<\gamma \mathrm{pi}$, ga-

18 La zona se entiende como el espacio geográfico en que se mueven pescadores y propietarios de centros de acopio, que aunque regularmente es un sitio particular de la costa, existirá un cambio en tal definición, cuando se estudia la modalidad de contactos en cadena o pirámide (formación de redes), que resulten pertenecer a otros sitios del litoral. 
rantizándose una mayor probabilidad de ser capturado y castigado en tal intercambio.

De igual forma, se tiene que:

$$
\text { DplP }<\text { DpiP }
$$

Con la expresión anterior, se refleja que en caso de ser sorprendido, capturando o transportando las especies, el pescador dedicado a la extracción ilegal del recurso, seguramente transportará una cantidad menor que quien se dedica a realizar pesca de forma apropiada. Además, quien se dedica a la ilegalidad, asume de forma equívoca que tiene cierto control sobre su actividad, situación que evidencia una mayor probabilidad de captura. Entonces:

$$
\gamma \text { plDplP }<\text { piDpiP }
$$

A pesar de la relación anterior, el hecho de tener $G=$ 0 implica que el propietario del centro de acopio en realidad no está dispuesto a pagar el cobro adicional por el recurso que ha sido extraído de forma ilegal, toda vez que tiene perfecto conocimiento de la permisividad en los controles por parte de la autoridad pública, así como, total conocimiento de la baja pertenencia que tiene la comunidad de pescadores por cumplir la norma social (pesca sustentable).

De otro lado, $\mathrm{Ppi}<\mathrm{Ppl}$ con lo que se observa que en este caso, el precio no tiene efecto sobre factores como la confidencialidad, porque el estatus social o la imagen ante el grupo de referencia, no importa. Así que no hay disposición a pagar dinero adicional por la actividad de pesca ilegal y, de esta manera, la captura ilegal de pesca puede ser óptima para el pescador teniendo en cuenta el escenario y la disposición a pagar por parte de los propietarios de los centros de acopio.

Ahora, estudiando la variable costos de transacción, se tiene que, aunque la captura ilegal implica, posiblemente, tener acuerdos con los otros pescadores de la zona, ello no implica desembolsos. Sin embargo, tal situación sí permite incluir el peligro que generan las actividades ilegales de captura alrededor de aquellas zonas que han sido definidas como sitios de reserva o áreas protegidas, como un costo, lo que posiblemente motive al pescador a intentar persuadir a la autoridad pública para desarrollar tranquilamente su actividad. Bajo el supuesto de que el pescador conoce la zona y ello le genera bajos niveles de riesgo, se puede afirmar que:

$$
\mathrm{Cpi}<\mathrm{Cpl}
$$

Proposición. El precio más bajo y el hecho de que $G$ $=0$ (lo que afecta la disposición a pagar) hace que el nivel de captura ilegal sea mayor y se tenga U $\pi \mathrm{pi}$ $\geq \mathrm{U} \pi \mathrm{pl}$. Adicionalmente, el conocimiento de que el castigo está limitado, tanto por el manejo de la zona, como por la permisividad de las leyes, permite que el óptimo de la pesca ilegal, dependa de la probabilidad de captura por parte de la autoridad. De esta manera, la pesca ilegal será óptima si:

$$
\begin{gathered}
1-\gamma \mathrm{piU} \pi \mathrm{pi}-1-\gamma \mathrm{plU} \pi \mathrm{pl} \geq \gamma \mathrm{plUDplP}- \\
\gamma \mathrm{piUDpiP} \geq 0
\end{gathered}
$$

La condición anterior establece que, siendo todo lo demás constante, un crecimiento en la probabilidad de captura por parte de la autoridad $\gamma$ pi podría hacer que la captura de pesca ilegal en el pacífico colombiano deje de ser rentable y con ello, se evidenciaría un respeto por parte de la comunidad, tanto por los controles de la autoridad, como por la norma social previamente establecida.

Por otra parte, si se supone que el conocimiento de la fragilidad de la norma hace que el castigo sea visto como insignificante, por lo tanto tiende a cero, la desigualdad (12), sería:

$$
\mathrm{U} \pi \mathrm{pi} \geq 1-\gamma \mathrm{pl} 1-\gamma \mathrm{pi} \mathrm{U} \pi \mathrm{pl}
$$

A partir de lo registrado en (13), queda claro que la optimalidad de realizar capturas inapropiadas del

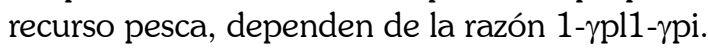

En el caso del propietario del centro de acopio, dado que la disposición a pagar por compra de especies provenientes de capturas ilegales o capturas legales es la misma cuando $G=0$, la decisión de compra está marcada totalmente por la probabilidad de captura por parte de la autoridad (entiéndase cierre o sellamiento del centro de acopio), con lo que se 
sugiere que el castigo debería ser lo suficientemente estricto, situación que como ya se explico anteriormente; no es así. Se entiende que normalmente el castigo consiste en simples contravenciones verbales y en una motivación por un cambio de conducta, en favor de la pesca sustentable.

\subsection{2. ¿Qué sucede cuando el status social importa?}

En este escenario se analiza el comportamiento de los agentes cuando $G=1$. Bajo este panorama, la utilidad esperada del pescador y propietario del centro de acopio, depende también de la probabilidad de ser recriminado y rechazado por el grupo social con el que convive.

El análisis de este escenario exige especificar los valores que puede tomar la función $\lambda \mathrm{i}$.

$$
\lambda \mathrm{i}=0 \text { si } \mathrm{f}=00,1 \text { si f1 si } \mathrm{f}=1 \in 0,1
$$

Adicionalmente, $\lambda$ pif $>\lambda$ plf $\quad \forall \mathrm{f} \in 0,1$.

Con la especificación de la función $\lambda$ y el supuesto de que UPpi-UPpl es continua en $f$ (de tal forma que es posible hacer uso del teorema del valor intermedio para funciones continuas), se establecen los siguientes resultados ${ }^{19}$ :

i. $\quad$ Si $f=0$ se regresa al caso $G=0$.

ii. Si $\mathrm{f} \in 0, \mathrm{f}^{*}$ sigue siendo cierto que UPpi $\geq \mathrm{UPpl}$ $\geq 0$, así que es óptimo realizar la pesca ilegal ${ }^{20}$.

iii. Si $\mathrm{f} \in$ se tiene $\mathrm{UPpl} \geq \mathrm{UPpi} \geq 0$, por lo que resulta óptimo el compromiso con la pesca legal y por tanto sustentable. iv. Si $f \in$ el incremento en la desutilidad hace que ninguna de las modalidades de pesca reporte beneficios positivos.

Los cuatro casos planteados se derivan de la forma de la función $\lambda \mathrm{i}$ para cada modalidad, lo que se constituye en un elemento fundamental para la existencia de los umbrales que limitan cada uno de los intervalos planteados.

La especificación de este modelo y la importancia otorgada a la variable $\lambda$ en la decisión tomada por los agentes, es coherente con lo planteado por Rodriguez-Sickert et al. (2008), pues dado el carácter de ilegal de la captura y extracción de distintas especies pertenecientes a la pesca, los agentes sienten mayor tranquilidad si el medio en que se desarrolla la misma es menos conservador ${ }^{21}$. De hecho, el último caso aquí planteado, revela que ante niveles de intolerancia elevados, lo que se puede traducir como grupos totalmente conservadores y que no soportan ningún acto que atente contra sus normas sociales ${ }^{22}$, los niveles de desutilidad para pescadores son tan severos, que ello se traduce en costos superiores a los beneficios y por tanto dedicarse a la pesca ilegal deja de ser atractivo.

Adicional a lo planteado anteriormente, resulta interesante destacar que los óptimos propuestos muestran que si la comunidad es en promedio una comunidad donde ese tipo de actos ilegales, y por tanto poco sustentables en términos de la preservación del recurso natural pesca, no se llevan a cabo, entonces la pesca ilegal hará que $\lambda \mathrm{i}$ sea muy grande, lo que afecta fuertemente la función de utilidad del individuo. Pero, si en promedio la comunidad (grupo de

19 Estos resultados se plantean suponiendo que se parte de unos niveles $\gamma$ pi y $\gamma$ pl que hacen que la pesca ilegal sea óptima. A partir de allí, se analiza que sucede con la utilidad esperada de cada modalidad si cambia solamente la variable $\lambda$ a partir de cambios en $\mathrm{f}$. Es evidente que cada vez que cambia ypi o ypl, la brecha entre la utilidad derivada de la pesca ilegal es distinta, por lo tanto, ello hará que los umbrales se muevan; pero manteniendo fijas las probabilidades de captura, es posible establecer los niveles de f necesarios para tener los resultados descritos.

20 Sin pérdida de generalidad, se asume que f* es la raíz de UPpi-UPpl.

21 Por medio conservador debe entenderse un espacio social en el que el grupo de referencia (especialmente comunidad de pescadores) no tolera actividades relacionadas con la pesca ilegal, es decir, individuos que se identifican exclusivamente con actividades de pesca sustentable.

22 Debe entenderse como acto fuera de la norma, una desviación de la media del comportamiento social. 
referencia) convive con ese y otro tipo de actos ilegales, $\lambda$ i será pequeño y ello pesará poco dentro de la función que mide la desutilidad ${ }^{23}$.

De otro lado, si $\lambda \mathrm{i}=0$ se retorna al caso en que la pesca ilegal es una actividad óptima, y si $\lambda \mathrm{i}=1$, es decir, si la comunidad no convive con esta ilegalidad, entonces lo mejor es no participar del intercambio directo de especies provenientes de una extracción de pesca ilegal.

Por último, el análisis de la decisión óptima del propietario del centro de acopio, implica tener en cuenta que, dado $\mathrm{G}=1$ se tiene: upi $<$ upl y Ppl $>$ Ppi , y que la disposición a pagar por el producto derivado de capturas legales (extracciones sustentables), es lo suficientemente alta como para que: upl - Ppl > upi - Ppi, con lo que el pescado legal, se constituye en su elección óptima, independientemente del valor que tome $\lambda \lambda$, si y solo si, el nivel de ff es inferior a $f^{24}$. En otras palabras, lo anterior es posible únicamente si la pesca legal le genera al propietario del centro de acopio un nivel de utilidad mayor, respecto a la utilidad que le derive la pesca ilegal.

\section{Comentarios finales}

El objetivo principal de este artículo es resaltar la importancia que puede llegar a tener un grupo de influencia, dentro de la toma de decisión racional de individuos que interactúan en el intercambio directo de especies provenientes de una pesca ilegal, en el pacífico colombiano. A partir de una propuesta metodológica de orden microeconómico, que responde a los supuestos de la elección racional económica esbozados en la escuela neoclásica de la economía del crimen, se analiza la problemática del intercambio directo de especies provenientes de una extracción de pesca ilegal en el pacífico colombiano, mediante el planteamiento de un juego no cooperativo con información completa.
El principal resultado establece que la interacción estratégica entre pescador y propietario del centro de acopio tiene un Equilibrio de Nash estable por acciones puras, en donde el pescador que se dedica a la captura de pesca ilegal, ante la opción revelada de compra por parte del propietario del centro de acopio, tiene como mejor respuesta el seguir realizando dicha actividad ilegal, y por tanto, se atenta a la sostenibilidad del recurso.

En tal sentido, una posible extensión de este documento, debería ser consistente con lo sugerido por Hansen et al. (2006), quienes afirman que para solucionar problemas de capturas de pesca ilegal, se deben diseñar mecanismos fiscales que tengan en cuenta procesos conjuntos de regulación externa, así como, métodos de autoregulación por parte de la comunidad de pescadores.

Ahora bien, dentro del otro posible equilibrio, en donde las estrategias son capturar el pescado de forma legal y comprar las especies provenientes de tal actividad sustentable, se destaca el supuesto implícito de que la comunidad no debería convivir con situaciones relacionadas con la pesca ilegal. Es decir, tanto para el propietario del centro de acopio, como para el pescador, sería mejor comprar y pescar en la legalidad, así esta modalidad implique mayores costos.

A partir de lo anterior, se debe comprender que el Equilibrio de Nash muestra estabilidad en el sentido de la valoración social que hacen los individuos inmersos en la interacción estratégica del intercambio directo de especies provenientes de una pesca ilegal, en el pacífico colombiano, de la siguiente forma: por un lado, el pescador debe tratar de vender más caro el pescado que ha sido capturado de forma ilegal, teniendo en cuenta el esfuerzo que le genera dicha actividad y que debe manejar un círculo de clientes

23 Este planteamiento es posible ajustarlo al de Brock \& Durlauf (2001) en el cual el individuo se ve castigado por desviarse del comportamiento que él espera sea el comportamiento promedio de su grupo referencial, ante una posible violación de la norma social, en este caso atentar contra la sostenibilidad del recurso natural de uso común: pesca.

24 Observando la ecuación que describe los pagos esperados para el propietario del centro de acopio, es posible darse cuenta que en este caso $U P_{p l}>U P_{p i}$. 
conocidos o referenciados, para evitar el riesgo de engaños que conduzcan a una captura y, por otro, el propietario del centro de acopio está dispuesto a comprar el recurso natural que ha sido capturado de forma ilegal, aún cuando tenga que pagar una tarifa adicional por utilizar esta modalidad. La razón es que ésta le brinda, sin duda, mayor posibilidad de obtener beneficios monetarios adicionales, siempre y cuando, mantenga una situación de confidencialidad en el intercambio.

Al margen de la propuesta metodológica que se esboza en este artículo, no se puede desconocer que actualmente existe gran preocupación a nivel mundial por el alto índice de especies pertenecientes a la pesca que están en riesgo de agotamiento. Esto es un indicio de la importancia del grupo de pares a la hora de asumir cierto tipo de conductas por parte de los individuos.

Es así, como a manera de sugerencia y aunque a través del artículo no se haga explícito, que la administración y autoridad pública debería preocuparse por el diseño de políticas públicas que realmente estén encaminadas a la preservación y sustentabilidad de los recursos naturales de uso común, como la pesca. En concreto, el legislador debería promover mecanismos efectivos de control, así como incentivos para quienes en realidad están en favor de las capturas y extracciones que contribuyen con la sustentabilidad de la pesca en el pacífico colombiano.

Finalmente, hacer un llamado de atención en el sentido de que aunque resulta más que importante combatir el problema del intercambio directo de especies provenientes de una pesca ilegal en el pacífico colombiano, tal situación no debe ser pensada solamente como un tema de represión y castigo; sino que además, se debe evidenciar que es un trabajo complejo e interdisciplinar, que exige concientización acerca del problema social que crea este mercado. Al mismo tiempo, se debe pensar en un proceso de integración en el que las poblaciones marginadas del pacífico colombiano se sientan parte de un proyecto de región, que les brinde entre otras oportunidades, unas condiciones dignas de empleo.

\section{Referencias}

Arrow, K. (1951). Alternative approaches to the theory of choice in risk-taking situations. Econometrica, 19:404-437.

Arroyo, S., Alegría, A., \& Bustamante, D. (2009). Economía del crimen y teoría de juegos: un modelo teórico del intercambio directo de drogas ilegales a pequeña escala. Anales de la Asociación Argentina de Economía Política. Disponible en: http://www.aaep.org.ar/anales/works/works2009/arroyo.pdf.

Baland, J. \& Platteau, J. (1997). Coordination problems in local-level resource management. Journal of Development Economics, 53(1):197-210

Becker, G. S. (1968). The Crime and Punishment: An Economic Approach. Journal of Political Economy, 76(2):169-217.

Bowles, S. (2008). Review of Microeconomics: Behavior, Institutions, and Evolution. Journal of Socio-Economics, 37(1):444-447.

Bowles, S. \& Gintis, H. (2002). Social capital and community governance. Economic Journal, 112:419-436.

Brock, W. \& Durlauf, S. (2001). Discrete Choice with Social Interactions. Review of Economic Studies, 68:235-260.

Cárdenas, J. (2003). Real wealth and experimental cooperation: Evidence from field experiments. Journal of Development Economics, 70 (2):263-289.

Cárdenas, J., Maya, D., \& López, M. (2003). Métodos experimentales y participativos para el análisis de la acción colectiva y la cooperación en el uso de recursos naturales por parte de comunidades rurales. Departamento de Desarrollo Rural y Regional. Facultad de Estudios Ambientales y Rurales. Pontificia Universidad Javeriana.

Cárdenas, J., Maya, D., \& López, M. (2002). Manglares, comunidad y cooperación. Pontificia Universidad Javeriana. WWF Colombia. Colgrafics Ltda.

Cárdenas, J. \& Ostrom, E. (2004). What do people bring into the game: experiments in the field about cooperation in the commons. Working papers 32, International Food Policy Research Institute (IFPRI), 32.

Casas, A., Cortez, D., \& Gamboa, L. (2003). Las comparaciones interpersonales y la evaluación de estados sociales alternativos. Revista Economía Institucional, 5 (08):147-160.

Ehrlich, I. (1996). Crime, Punishment, and the Market for Offenses. The Journal of Economic Perspectives, 10(1):43-67.

Elbers, C., Lanjouw, P., Mistiaen, J., Ôzler, B., \& Simler, K. (2004). On the Inequal Inequalities of Poor Communities. The World Bank Economic Review, 18(3) Oxford, Reino Unido: Oxford University Press:401-421.

Hansen, L.-G., Jensen, F., Brandt, U.-S., \& Vestergaard, N. (2006). Illegal landings: an aggregate catch self-reporting mechanism. American Journal Agricultural Economics, 88(4):974-985.

Hardin, G. (1968). The tragedy of the Commons. Science, 162:1243-1248.

Harsanyi, J. (1955). Cardinal Welfare, Individualistic Ethics, and Interpersonal Comparisons of Utility. The Journal of Political Ecomomy, 63:309-321.

Hatcher, A., Jaffry, S., Thebaud, O., \& Bennett, E. (2000). Normative and Social Influences Affecting Compliance with Fishery Regulations. Land Economics, 76 (3):448-461.

Jensen, F. y Vestergaard, N. (2000). Moral Hazard Problems in Fisheries Regulation: The Case of Illegal Landings. Society, Economics and the Environments, 9:7-19. 
Milliman, S. (1986). Optimal Fishery Management in the Presence of Illegal Activity. Journal of Environmental Economics and Management, 13:363-387.

Neumann, V. \& Morgenstern, O. (1947). Theory of Games and Economic Behavior. Princenton University Press.

Nussbaum, M. \& Sen, A. (1996). La calidad de vida. The United Nations University.

Ostrom, E. (1990). Governing the Commons: The Evolution of Institutions for Collective Action. Cambridge.

Ostrom, E. (2000). El gobierno de los comunes. Fondo de Cultura Económica.

Ostrom, E., Gardner, R., \& Walker, J. (1994). Rules, Games, and Common-Pool Resources. Ann Arbor.
Rodriguez-Sickert, C., Guzmán, R., \& Cárdenas, J. (2008). Institutions influence preferences: Evidence from a common pool resource experiment. Journal of Economic Behavior \& Organization, 67:215-227.

Sen, A. (1988). The Concept of Development. In Handbook of Development Economics. Cap. I, Vol. I, Amsterdam, North Holland.

Sugden, R. (1984). Reciprocity: The Supply of Public Goods through Voluntary Contributions. Economic Journal, Royal Economic Society, 94(376):772-787.

Trisak, J. (2005). Applying game theory to analyze the influence of biological characteristics on fishers cooperation in fisheries co-management. Fisheries Research, 75:164-174. 